\title{
Generalized Parton Distributions from charged current meson production
}

\author{
Ivan Schmidt, Marat Siddikov*, \\ ${ }^{1}$ Departamento de Física, Universidad Técnica Federico Santa María, \\ y Centro Científico - Tecnológico de Valparaíso, Casilla 110-V, Valparaíso, Chile \\ E-mail: Ivan.Schmidteusm.cl, Marat.Siddikov@usm.cl
}

We suggest that charged current production of mesons in Bjorken kinematics allows a very clean extraction of GPDs, and demonstrate that simultaneous study of parity conjugate mesons (e.g. $\rho$-mesons and pions) provides very sensitive probes of the difference of meson distribution amplitudes. We expect that such processes might be studied in the future in neutrino-induced and electron-induced processes.

PACS: $13.15 .+9,13.85 .-t$

European Physical Society Conference on High Energy Physics - EPS-HEP2019 -

10-17 July, 2019

Ghent, Belgium

${ }^{*}$ Speaker. 


\section{Introduction}

Understanding the structure of the nucleon remains a challenging problem which is being actively studied by both experimental and theoretical research groups. It has been established that for certain processes in Bjorken kinematics $[1,2]$ this structure effectively reduces to the so-called generalized parton distributions (GPDs), which encode information about the parton-level scattering amplitudes. The early studies of GPDs focused on deeply virtual Compton scattering (DVCS) [3] and deeply virtual meson production (DVMP) $[4,5,6]$. However, nowadays it is understood that in the kinematics of modern high luminosity experiments used for study of exclusive processes, the Bjorken limit results might suffer from large higher twist and next-to-leading order corrections $[6,7,8]$. These corrections are expected of decrease as a function of the virtuality $Q^{2}$ passed to hadron. However, a significant increase of $Q^{2}$ is not an experimentally viable option, since the cross-sections of exclusive processes decrease rapidly as $\sim 1 / Q^{2 n}$ with $n \gtrsim 2-3$. For this reason, the analysis of GPDs should be based on global self-consistent analysis of various channels [9], and the list of processes currently used for the analysis should be significantly extended.

We suggest that the program of GPD studies could be complemented by analysis of Charged Current DVMP (CCDVMP), which could be measured either in neutrino-induced or in electroninduced processes. Such channels on unpolarized target get the dominant contribution from the GPDs $H_{u}, H_{d}$ and have a small uncertainty due to twist-3 effects [10]. Due to $V-A$ structure of the charged current, in the leading twist the CCDVMP cross-sections of parity conjugate mesons are sensitive to exactly the same set of GPDs, which permits various consistency checks: as we will demonstrate below, this gives a sensitive probe to study the difference of distribution amplitudes of meson pairs.

The paper is structured as follows: In the following Section 2 we discuss the framework used for evaluation of meson production, taking into account NLO and some of the higher twistcorrections. In Section 3 we present numerical results and draw conclusions. Due to space limitations, in this proceeding we omitted some technical details which might be found in our recent [9].

\section{CCDVMP cross-section in the Bjorken limit}

The CCDVMP might be studied both in neutrino-induced and electron-induced processes. For the sake of definiteness, in what follows we will consider the case of electroproduction, $e p \rightarrow$ $v_{e} M p$. The cross-section of the process in this case is given by

$$
\frac{d \sigma}{d t d x_{B} d Q^{2}}=\frac{G_{F}^{2} x_{B}^{2}\left(1-y-\frac{\gamma^{2} y^{2}}{4}\right)}{64 \pi^{3} Q^{2}\left(1+Q^{2} / M_{W}^{2}\right)^{2}\left(1+\gamma^{2}\right)^{3 / 2}} \sum_{v v^{\prime}} \mathscr{A}_{v^{\prime}, v L^{\prime}}^{*} \mathscr{A}_{\nu^{\prime}, v L},
$$

where $t=\left(p_{2}-p_{1}\right)^{2}$ is the momentum transfer to the proton, $Q^{2}=-q^{2}$ is the virtuality of the charged boson, $x_{B}=Q^{2} /(2 p \cdot q)$ is the Bjorken variable, $E_{e}$ is the energy of the projectile electron in the target rest frame, $M_{W}$ is the mass of the heavy bosons $W^{ \pm}, G_{F}$ is the Fermi constant; the subscript indices $v$ and $v^{\prime}$ in the amplitude $\mathscr{A}$ refer to helicity states of the baryon before and after interaction, and the letter $L$ reflects the fact that in the Bjorken limit the dominant contribution comes from the longitudinally polarized massive bosons $W^{ \pm}[1,2]$, and we also used shorthand 
notations

$$
\gamma=\frac{2 m_{N} x_{B}}{Q}, \quad y=\frac{Q^{2}}{s_{e p} x_{B}}=\frac{Q^{2}}{2 m_{N} E_{e} x_{B}} .
$$

In Bjorken kinematics, the amplitude $\mathscr{A}_{v^{\prime}, v L}$ factorizes into convolution of hard and soft parts,

$$
\mathscr{A}_{\nu^{\prime}, v}=\int_{-1}^{+1} d x \sum_{q=u, d, s, g} \sum_{\lambda \lambda^{\prime}} \mathscr{H}_{v^{\prime} \lambda^{\prime}, v \lambda}^{q} \mathscr{C}_{\lambda \lambda^{\prime}}^{q}
$$

where $x$ is the average light-cone fraction of the parton, superscript $q$ is its flavor, $\lambda$ and $\lambda^{\prime}$ are the helicities of the initial and final partons. The soft matrix element $\mathscr{H}_{v^{\prime} \lambda^{\prime}, v \lambda}^{q}$ in (2.3) is diagonal in quark helicities $\left(\lambda, \lambda^{\prime}\right)$, and for the twist-2 GPDs has a form

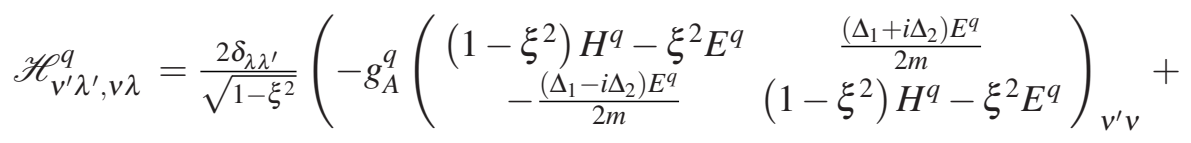

$$
\begin{aligned}
& \left.+\operatorname{sgn}(\lambda) g_{V}^{q}\left(\begin{array}{cc}
-\left(1-\xi^{2}\right) \tilde{H}^{q}+\xi^{2} \tilde{E}^{q} & \frac{\left(\Delta_{1}+i \Delta_{2}\right) \xi \tilde{E}^{q}}{2 m} \\
\frac{\left(\Delta_{1}-i \Delta_{2}\right) \xi \tilde{E}^{q}}{2 m} & \left(1-\xi^{2}\right) \tilde{H}^{q}-\xi^{2} \tilde{E}^{q}
\end{array}\right)_{v^{\prime} v}\right)
\end{aligned}
$$

where the constants $g_{V}^{q}, g_{A}^{q}$ are the vector and axial current couplings to quarks; we use standard notation for the leading twist GPDs $H^{q}, E^{q}, \tilde{H}^{q}$ and $\tilde{E}^{q}[5,6,9]$. The evaluation of the structure function $\mathscr{C}_{\lambda \lambda^{\prime}}^{q}$ is quite straightforward and might be found in [9] for different channels. For the processes in which baryon does not change its internal state, there are additional contributions from gluon GPDs which might be important at high energies.

The quark distribution amplitudes of pseudoscalar and longitudinally polarized vector mesons have a structure which differs only by $\gamma_{5}$ matrix in quark-antiquark operators (modulo some trivial numerical prefactor) $[11,12,13]$. In case of the photoproduction, due to the aforementioned difference, the leading twist amplitudes are sensitive to complementary sets of GPDs. In case of charged-current meson production, due to $V-A$ structure of the weak interaction the amplitudes are sensitive to the same set of GPDs, and the amplitudes of both processes are related by a mere substitution

$$
f_{\pi} \phi_{2 ; \pi}(\alpha) \leftrightarrow f_{\rho_{L}} \phi_{2, \rho}^{(\|)}(\alpha)
$$

where $\phi_{2 ; M}$ and $f_{M}$ are the distribution amplitudes and decay constants of the corresponding meson $\left(M=\rho_{L}^{ \pm}, \pi^{ \pm}\right)$. In the leading order over $\alpha_{s}$, the distribution amplitude contributes to the coefficient function multiplicatively as a minus-first moment $\left\langle\phi_{2, M}^{-1}\right\rangle$. For this reason we may expect that the ratio of the cross-sections of the longitudinally polarized $\rho^{ \pm}$and $\pi^{ \pm}$mesons

$$
R_{\rho / \pi}\left(x_{B}, Q^{2}\right)=\frac{d \sigma_{W^{ \pm} p \rightarrow \rho_{L}^{ \pm} p}}{d \sigma_{W^{ \pm} p \rightarrow \pi^{ \pm} p}} \approx \mathrm{const},
$$

should not depend on GPDs of the target, being the same for proton, neutron and nuclear targets. In the Bjorken kinematics the twist-two distributions $\phi_{2 ; \pi}, \phi_{2, \rho}^{(\|)}$might be decomposed as

$$
\phi_{2}\left(z, \mu^{2}\right)=6 z(1-z)\left(1+\sum_{n>0} a_{2 n}\left(\mu^{2}\right) C_{2 n}^{3 / 2}(2 z-1)\right),
$$


where the coefficients $a_{2 n}\left(\mu^{2}\right)$ have mild multiplicative dependence on the factorization scale $\mu$ and are frequently used to characterize the shape of the distribution amplitude. At present it is expected that the coefficients $a_{2 n}$ should be small, with current estimates [14, 15]

$$
\left|a_{2}\left(\mu^{2} \approx 2 \mathrm{GeV}^{2}\right)\right| \sim\left|a_{4}\left(\mu^{2} \approx 2 \mathrm{GeV}^{2}\right)\right| \lesssim 0.1, \quad a_{2 n} \approx 0 \quad \text { for } n \geq 3 .
$$

In terms of the expansion coefficients $a_{2 n}$ defined in (2.7), the moments may be evaluated exactly and are given by $\left\langle\phi_{2}^{-1}\right\rangle=1+\sum_{n} a_{2 n}$, so the ratio (2.6) is given by

$$
R_{\rho / \pi}^{(\mathrm{LO}, \mathrm{tw}-2)} \approx\left(f_{\rho} / f_{\pi}\right)^{2}\left(\frac{1+\sum a_{2 n, \rho}^{(\|)}}{1+\sum a_{2 n, \pi}}\right)^{2} \approx \frac{f_{\rho}^{2}}{f_{\pi}^{2}}\left[1+2 \sum_{n>0} r_{2 n}\left(a_{2 n, \rho}^{(\|)}-a_{2 n, \pi}\right)+\mathscr{O}\left(\left(a_{2, \rho}^{(\|)}-a_{2, \pi}\right)^{2}\right)\right] .
$$

In this order over $\alpha_{s}$ all the expansion coefficients $r_{2 n}$ defined in (2.9) equal to unity, $r_{2 n}\left(x_{B}, Q^{2}\right)=$ 1 , and do not depend on $\left(x_{B}, Q^{2}\right)$. The next-to-leading order corrections $\delta r_{2 n} \sim \mathscr{O}\left(\alpha_{s}\right)$ introduce a mild dependence on $\left(x_{B}, Q^{2}\right)$ (see [9] for more details). In contrast, the contributions of the twist3 corrections introduce a strong $\sim 1 / Q^{2}$ dependence which could be clearly distinguished from experimental data.

\section{Results and discussion}

For the sake of definiteness, for numerical estimates we use the Kroll-Goloskokov parametrization of GPDs $[16,7]$. We will start discussion assuming the dominance of the twist two contribution, and assuming the asymptotic form for meson distribution amplitudes. In this case the difference between pion and $\rho$-meson cross-sections exists only due to "kinematic" higher twist effects $\sim \mathscr{O}\left(M_{\pi, \rho}^{2} / Q^{2}\right)$ and is negligible.

In the Figure 1 we show the differential cross-section $d \sigma / d x_{B} d Q^{2}$ for charged meson $\left(\rho^{-}, \pi^{-}\right)$ production in JLab kinematics (left panel) and Electron Ion Collider (right panel, $\sqrt{s_{e p}} \approx 100$ $\mathrm{GeV}$ assumed). At fixed electron energy $E_{e}$ and virtuality $Q^{2}$, the cross-section as a function of $x_{B}$ has a typical bump-like shape, which is explained by an interplay of two factors. For small $x_{B} \sim Q^{2} / 2 m_{N} E_{e}$ the elasticity $y$ defined in (2.2) approaches one, which causes a suppression due to a kinematic prefactor in (2.1). In the opposite limit, the suppression $\sim(1-x)^{n}$ is due to the $x \rightarrow 1$ behavior of the parton distributions. In evaluation of the coefficient function we take into account NLO corrections, which give a sizeable contribution for $Q^{2} \lesssim 10 \mathrm{GeV}^{2}$. The band around the curves reflects the theoretical uncertainty due to higher order corrections, which was obtained varying the factorization scale $\mu_{F}$ in the range $\mu_{F} \in(Q / 2,2 Q)$ (see $[6,16,7,17,18]$ for more details). For JLab kinematics (right plot) the dominant contribution comes from the GPDs $H^{u}, H^{d}$, whereas in case of EIC there is a sizeable contribution from gluon GPDs.

In order to quantize the sensitivity of the cross-section to deviation of meson DA from asymptotic form, in the Figure 2 we have shown the dependence of the first two coefficients $r_{2}\left(x_{B}, Q^{2}\right)$ and $r_{4}\left(x_{B}, Q^{2}\right)$ defined in (2.6) as a function of $x_{B}$ and $Q^{2}$. These coefficients do not depend on energy of the electron beam $E$ because at fixed $\left(x_{B}, Q^{2}\right)$ the dependence on $E$ contributes only via a common $y$-dependent prefactor in (2.1), and does not contribute to $r_{2 n}$. The dependence of $r_{2 n}$ on $Q^{2}$ is very mild and is due to logarithmic dependence of running coupling in the NLO contribution and evolution of parton distributions. The dependence of $r_{2 n}$ on $x_{B}$ exists due to different 

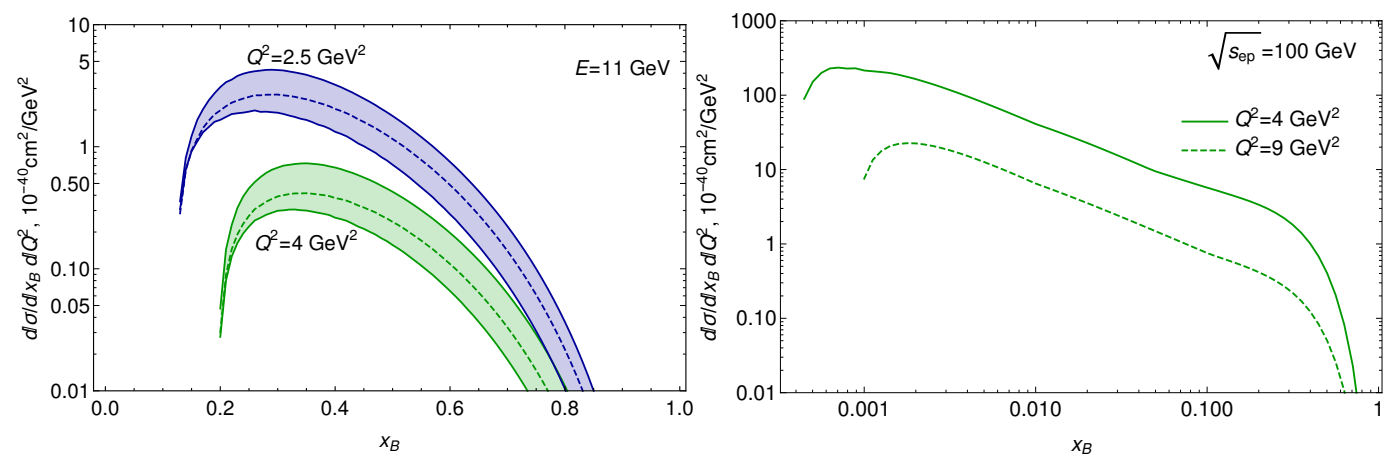

Figure 1: (color online) Left plot: Charged current meson production cross-section on proton target in JLab kinematics (fixed electron energy $E=11 \mathrm{GeV}$ ). Evaluations are performed using NLO coefficient functions, as discussed in Section 2. The width of the band represents the uncertainty due to the factorization scale choice $\mu_{F} \in(Q / 2,2 Q)$, as explained in the text. Right plot: $x_{B}$-dependence of the cross-section in EIC kinematics with $\sqrt{s}_{e p} \approx 100 \mathrm{GeV}$.

$x_{B}$-dependence of the leading order and next-to-lading order coefficient functions. The latter fact implies that the ratio of the cross-sections (2.6) has a mild dependence on $\left(x_{B}, Q^{2}\right)$, and its value is almost entirely determined by the values of parameters

$$
a_{2}=a_{2, \rho}^{(\|)}-a_{2, \pi}, \quad a_{4}=a_{4, \rho}^{(\|)}-a_{4, \pi} .
$$

As could be seen from the Figure 2, for the currently expected phenomenological values of parameters $a_{2}, a_{4}$ in the range (2.8) the ratio (2.6) might change up to $20 \%$. Given that the functions $r_{2}\left(x, Q^{2}\right), r_{4}\left(x, Q^{2}\right)$ are known, experimental measurement of $R_{\rho / \pi}$ in sufficiently large kinematic range could allow us to extract separately the values of $a_{2}$ and $a_{4}$.
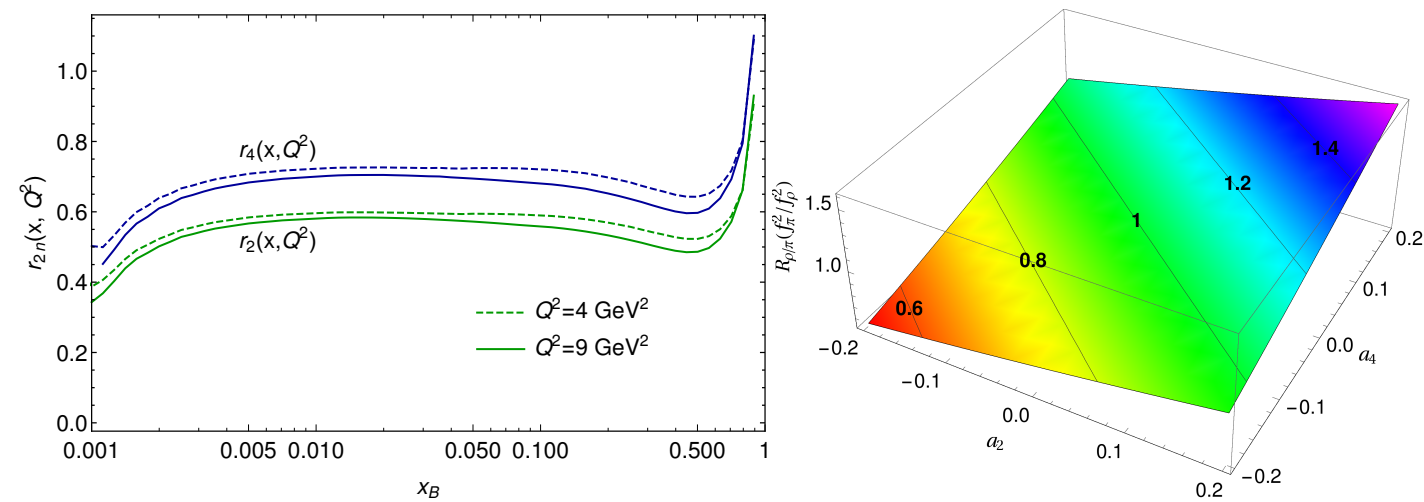

Figure 2: (color online) Left: Values of the coefficients $r_{2 n}\left(x_{B}, Q^{2}\right)$. The two bottom curves correspond to $r_{2}\left(x_{B}, Q^{2}\right)$; the two upper curves correspond to $r_{4}\left(x_{B}, Q^{2}\right)$. For both cases dashed lines correspond to $Q^{2}=4 \mathrm{GeV}^{2}$, solid lines correspond to $Q^{2}=9 \mathrm{GeV}^{2}$. All evaluations performed with account of NLO correction. See the text for more explanations of the behaviour of the curves. Right: expected value of the variable $R_{\rho / \pi}\left(f_{\pi} / f_{\rho}\right)^{2}$ as a function of possible values of $a_{2}$ and $a_{4}$ for $x_{B}=0.1$ and $Q^{2}=4 \mathrm{GeV}^{2}$. For the case of asymptotic form distributions of both mesons $\left(a_{2}=a_{4}=0\right)$ the variable $R_{\rho / \pi}\left(f_{\pi} / f_{\rho}\right)^{2}=1$.

As was discussed in detail in [9], the uncertainty due to twist three harmonics is small and 
does not exceed ten per cent in the kinematics of interest. For this reason we expect that these corrections will not affect significantly the ratio $R\left(a_{2}, a_{4}\right)$ shown in the right panel of the Figure 2 .

To summarize, we suggested to use the CCDVMP processes for a very clean extraction of GPDs of the nucleons and estimated the cross-sections in different kinematics. We found that the parity conjugate mesons (e.g. $\rho_{L}^{ \pm}$and $\pi^{ \pm}$) are sensitive to the same set of GPDs, for this reason the ratio of their cross-sections (2.6) gives possibility to probe the difference of the distribution amplitudes. We expect that this ratio should be constant in the leading order, yet acquires a mild dependence on $\left(x_{B}, Q^{2}\right)$ due to NLO corrections. We expect that the CCDVMP processes might be studied either in the future neutrino-induced experiments or in electron-induced experiments.

\section{Acknowledgments}

This research was partially supported by Proyecto Basal FB 0821 (Chile), the Fondecyt (Chile) grant 1180232 and CONICYT (Chile) grant PIA ACT1413.

\section{References}

[1] X. D. Ji and J. Osborne, Phys. Rev. D 58 (1998) 094018 [arXiv:hep-ph/9801260].

[2] J. C. Collins and A. Freund, Phys. Rev. D 59, 074009 (1999).

[3] R. Dupré, M. Guidal, S. Niccolai and M. Vanderhaeghen, arXiv:1704.07330 [hep-ph].

[4] D. Mueller, D. Robaschik, B. Geyer, F. M. Dittes and J. Horejsi, Fortsch. Phys. 42, 101 (1994) [arXiv:hep-ph/9812448].

[5] K. Goeke, M. V. Polyakov and M. Vanderhaeghen, Prog. Part. Nucl. Phys. 47, 401 (2001) [arXiv:hep-ph/0106012].

[6] M. Diehl, Phys. Rept. 388, 41 (2003) [arXiv:hep-ph/0307382].

[7] S. V. Goloskokov and P. Kroll, Eur. Phys. J. A 47, 112 (2011) [arXiv:1106.4897 [hep-ph]].

[8] G. R. Goldstein, J. O. G. Hernandez and S. Liuti, arXiv:1201.6088 [hep-ph].

[9] M. Siddikov, I. Schmidt, Phys.Rev. D 99, 116005 (2019) [arXiv: 1904.04252 [hep-ph]]

[10] B. Z. Kopeliovich, I. Schmidt and M. Siddikov, Phys. Rev. D 89, no. 5, 053001 (2014) [arXiv:1401.1547 [hep-ph]].

[11] P. Ball, V. M. Braun and A. Lenz, JHEP 0605 (2006) 004 [arXiv:hep-ph/0603063].

[12] P. Ball, V. M. Braun, Y. Koike and K. Tanaka, Nucl. Phys. B 529, 323 (1998) [hep-ph/9802299].

[13] B. Z. Kopeliovich, I. Schmidt and M. Siddikov, Nucl. Phys. A 918, 41 (2013) [arXiv:1108.5654 [hep-ph]].

[14] H. B. Fu, X. G. Wu, W. Cheng and T. Zhong, Phys. Rev. D 94, no. 7, 074004 (2016) [arXiv:1607.04937 [hep-ph]].

[15] G. S. Bali et al., Eur. Phys. J. C 78, no. 3, 217 (2018) [arXiv:1709.04325 [hep-lat]].

[16] S. V. Goloskokov and P. Kroll, Eur. Phys. J. C 65, 137 (2010) [arXiv:0906.0460 [hep-ph]].

[17] M. Diehl and W. Kugler, Eur. Phys. J. C 52, 933 (2007) [arXiv:0708.1121 [hep-ph]].

[18] B. Pire, L. Szymanowski and J. Wagner, Phys. Rev. D 95, no. 9, 094001 (2017) [arXiv:1702.00316 [hep-ph]]. 